\title{
Effective multi-Higgs couplings to gluons
}

\section{Michael Spira}

Paul Scherrer Institut, CH-5232 Villigen PSI, Switzerland

E-mail: michael.spira@psi.ch

\begin{abstract}
Standard-Model Higgs bosons are dominantly produced via the gluon-fusion mechanism $g g \rightarrow H$ at the LHC, i.e. in a loop-mediated process with top loops providing the dominant contribution. For the measured Higgs boson mass of $\sim 125 \mathrm{GeV}$ the limit of heavy top quarks provides a reliable approximation as long as the relative QCD corrections are scaled with the full mass-dependent LO cross section. In this limit the Higgs coupling to gluons can be described by an effective Lagrangian. The same approach can also be applied to the coupling of more than one Higgs boson to gluons. We will derive the effective Lagrangian for multi-Higgs couplings to gluons up to $\mathrm{N}^{4} \mathrm{LO}$ thus extending previous results for more than one Higgs boson. Moreover we discuss gluonic Higgs couplings up to NNLO, if several heavy quarks contribute.
\end{abstract}

KEYwORDS: NLO Computations

ARXIV EPRINT: 1607.05548 


\section{Contents}

1 Introduction 1

2 Standard-model Higgs bosons 2

$\begin{array}{lll}3 & \text { Several heavy quarks } & 9\end{array}$

4 Conclusions $\quad 10$

\section{Introduction}

The discovery of a resonance with $125 \mathrm{GeV}$ mass $[1,2]$ that is compatible with the StandardModel (SM) Higgs boson [3] marked a milestone in particle physics. The existence of the Higgs boson is inherently related to the mechanism of spontaneous symmetry breaking [4-8] while preserving the full gauge symmetry and the renormalizability of the SM $[9,10]$. The dominant production process of the Higgs boson at the LHC is the loop-induced gluonfusion process mediated by top-quark loops and to a lesser extent bottom- and charmquark loops [11]. The QCD corrections are known up to $\mathrm{N}^{3} \mathrm{LO}$ in the limit of heavy top quarks [12-27], while the full quark mass dependence is only known up to NLO [28-31]. At NNLO subleading terms in the large top mass expansion [32-35] and leading contributions to the top+bottom interference [36] are known. The limit of heavy top quarks has also been adopted for threshold-resummed calculations [37-48], while the inclusion of finite quarkmass effects in the resummation has been considered recently [49-51]. It has been shown that the limit of heavy top quarks $m_{t}^{2} \gg M_{H}^{2}$ provides a reasonable approximation to the calculation of the gluon-fusion cross section with full mass dependence as long as the relative QCD corrections are scaled with the fully massive LO cross section [28, 37, 38]. In the heavy-top-quark limit the calculation of the gluon-fusion cross section can be simplified by starting from an effective Lagrangian describing the Higgs coupling to gluons after integrating out the top contribution [52-54]. The same approach has also been applied to Higgs pair production via gluon fusion, $g g \rightarrow H H$, at NLO [55], NNLO [56-58] as well as to threshold resummation up to NNLL $[59,60]$. It has been shown that finite mass effects amount to about $5 \%$ in the single Higgs case and $15 \%$ for Higgs boson pairs [61-65].

In this letter we will derive the effective Lagrangian for multi-Higgs couplings to gluons to $\mathrm{N}^{4} \mathrm{LO}$ for arbitrary numbers of external Higgs bosons thus extending previous work beyond the single-Higgs case. In section 2 we will discuss and present the effective Lagrangian for the SM Higgs boson up to $\mathrm{N}^{4} \mathrm{LO}$, while section 3 will extend this analysis to an arbitrary number of heavy quarks contributing to the gluonic Higgs coupling up to NNLO. In section 4 we will conclude. 


\section{Standard-model Higgs bosons}

The starting point for the derivation of the effective Lagrangian in the heavy-top-quark limit is the low-energy limit of the top-quark contributions to the Wilson coefficient of the gluonic field-strength operator $\hat{G}^{a \mu \nu} \hat{G}_{\mu \nu}^{a}$, where $\hat{G}^{a \mu \nu}$ denotes the ( $\overline{\mathrm{MS}}$-subtracted) gluonic operator of colour-SU(3) in the low-energy limit with 6 active flavours, ${ }^{1}$

$$
\mathcal{L}_{g}=-\frac{1-\Pi_{t}}{4} \hat{G}^{a \mu \nu} \hat{G}_{\mu \nu}^{a}
$$

The Wilson coefficient $\Pi_{t}$ denotes the gauge-invariant vacuum polarization function of the gluon that is determined by the top-quark contribution to the gluon self-energy and the two-point-function parts of the external vertices attached to the gluons. This boils down to the inverse top-quark contribution to the strong coupling constant so that $\Pi_{t}$ is related to the decoupling relation between the strong coupling constant in an $\left(N_{F}+1\right)$ and $N_{F}$-flavour theory $\left(N_{F}=5\right)$,

$$
\alpha_{s}^{\left(N_{F}\right)}\left(\mu_{R}^{2}\right)=\zeta_{\alpha_{s}} \alpha_{s}^{\left(N_{F}+1\right)}\left(\mu_{R}^{2}\right), \quad \zeta_{\alpha_{s}}=1+\sum_{n} D_{n}\left(\frac{\alpha_{s}^{\left(N_{F}+1\right)}\left(\mu_{R}^{2}\right)}{\pi}\right)^{n}
$$

with the perturbative coefficients up to fourth order [66-69] $\left[L_{t}=\log \left(\mu_{R}^{2} /{\overline{m_{t}}}^{2}\left(\mu_{R}^{2}\right)\right)\right]$

$$
\begin{aligned}
D_{1}= & -\frac{1}{6} L_{t} \quad D_{2}=\frac{11}{72}-\frac{11}{24} L_{t}+\frac{1}{36} L_{t}^{2} \\
D_{3}= & \frac{564731}{124416}-\frac{82043}{27648} \zeta_{3}-\frac{2633}{31104} N_{F}-\frac{955-67 N_{F}}{576} L_{t}+\frac{53-16 N_{F}}{576} L_{t}^{2}-\frac{1}{216} L_{t}^{3} \\
D_{4}= & \frac{291716893}{6123600}-\frac{121}{4320} \log ^{5} 2+\frac{3031309}{1306368} \log ^{4} 2+\frac{121}{432} \zeta_{2} \log ^{3} 2-\frac{3031309}{217728} \zeta_{2} \log ^{2} 2 \\
& +\frac{2057}{576} \zeta_{4} \log 2+\frac{1389}{256} \zeta_{5}-\frac{76940219}{2177280} \zeta_{4}-\frac{2362581983}{87091200} \zeta_{3}+\frac{3031309}{54432} a_{4}+\frac{121}{36} a_{5} \\
& -\frac{151369}{2177280} X_{0}+N_{F}\left(-\frac{4770941}{2239488}+\frac{685}{124416} \log ^{4} 2-\frac{685}{20736} \zeta_{2} \log ^{2} 2+\frac{3645913}{995328} \zeta_{3}\right. \\
& \left.-\frac{541549}{165888} \zeta_{4}+\frac{115}{576} \zeta_{5}+\frac{685}{5184} a_{4}\right)+N_{F}^{2}\left(-\frac{271883}{4478976}+\frac{167}{5184} \zeta_{3}\right) \\
& -\left[\frac{7391699}{746496}+\frac{2529743}{165888} \zeta_{3}+N_{F}\left(\frac{110341}{373248}-\frac{110779}{82944} \zeta_{3}\right)-N_{F}^{2} \frac{6865}{186624}\right] L_{t} \\
& +\left(\frac{2177}{3456}-N_{F} \frac{1483}{10368}-N_{F}^{2} \frac{77}{20736}\right) L_{t}^{2}-\left(\frac{1883}{10368}+N_{F} \frac{127}{5184}-\frac{N_{F}^{2}}{324}\right) L_{t}^{3}+\frac{L_{t}^{4}}{1296}
\end{aligned}
$$

\left. where ${\overline{m_{t}}}^{2}\left(\mu_{R}^{2}\right)\right)$ denotes the $\overline{\mathrm{MS}}$ top mass at the renormalization scale $\mu_{R}$. The constants used in this expression are given by $a_{n}=L i_{n}(1 / 2)$ and $X_{0}=1.8088795462 \ldots$ (only known numerically) [70]. The decoupling coefficient contains one-particle-reducible contributions and the Wilson coefficient of the Lagrangian eq. (2.1) is obtained from the inverse,

$$
\Pi_{t}=1-\frac{1}{\zeta_{\alpha_{s}}}=\sum_{n} C_{n}\left(\frac{\alpha_{s}^{\left(N_{F}+1\right)}}{\pi}\right)^{n}
$$

\footnotetext{
${ }^{1}$ The same ansatz has also been used in the derivation of the effective $\mathrm{Hgg}$ coupling in refs. [37, 38].
} 
with the perturbative coefficients up to fifth order

$$
\begin{aligned}
& C_{1}=-\frac{1}{6} L_{t} \quad C_{2}=\frac{11}{72}-\frac{11}{24} L_{t} \\
& C_{3}=\frac{564731}{124416}-\frac{82043}{27648} \zeta_{3}-\frac{2633}{31104} N_{F}-\frac{2777-201 N_{F}}{1728} L_{t}-\frac{35+16 N_{F}}{576} L_{t}^{2} \\
& C_{4}=\frac{1166295847}{24494400}-\frac{121}{4320} \log ^{5} 2+\frac{3031309}{1306368} \log ^{4} 2+\frac{121}{432} \zeta_{2} \log ^{3} 2-\frac{3031309}{217728} \zeta_{2} \log ^{2} 2 \\
& +\frac{2057}{576} \zeta_{4} \log 2+\frac{1389}{256} \zeta_{5}-\frac{76940219}{2177280} \zeta_{4}-\frac{2362581983}{87091200} \zeta_{3}+\frac{3031309}{54432} a_{4}+\frac{121}{36} a_{5} \\
& -\frac{151369}{2177280} X_{0}+N_{F}\left(-\frac{4770941}{2239488}+\frac{685}{124416} \log ^{4} 2-\frac{685}{20736} \zeta_{2} \log ^{2} 2+\frac{3645913}{995328} \zeta_{3}\right. \\
& \left.-\frac{541549}{165888} \zeta_{4}+\frac{115}{576} \zeta_{5}+\frac{685}{5184} a_{4}\right)+N_{F}^{2}\left(-\frac{271883}{4478976}+\frac{167}{5184} \zeta_{3}\right) \\
& +\left[\frac{2875235}{248832}-\frac{897943}{55296} \zeta_{3}-N_{F}\left(\frac{40291}{124416}-\frac{110779}{82944} \zeta_{3}\right)+N_{F}^{2} \frac{6865}{186624}\right] L_{t} \\
& -\left(\frac{1333}{10368}+N_{F} \frac{1081}{10368}+N_{F}^{2} \frac{77}{20736}\right) L_{t}^{2}-\left(\frac{1697}{10368}+N_{F} \frac{175}{5184}-N_{F}^{2} \frac{1}{324}\right) L_{t}^{3} \\
& C_{5}=C_{50}+\left(-\frac{685}{10368} N_{F}^{2} a_{4}-\frac{11679301}{435456} N_{F} a_{4}+\frac{93970579}{217728} a_{4}-\frac{121}{72} N_{F} a_{5}+\frac{3751}{144} a_{5}\right. \\
& +\frac{121}{8640} N_{F} \log ^{5} 2-\frac{3751}{17280} \log ^{5} 2-\frac{685}{248832} N_{F}^{2} \log ^{4} 2-\frac{11679301}{10450944} N_{F} \log ^{4} 2 \\
& +\frac{93970579}{5225472} \log ^{4} 2-\frac{121}{864} N_{F} \zeta_{2} \log ^{3} 2+\frac{3751}{1728} \zeta_{2} \log ^{3} 2+\frac{685}{41472} N_{F}^{2} \zeta_{2} \log ^{2} 2 \\
& +\frac{11679301}{1741824} N_{F} \zeta_{2} \log ^{2} 2-\frac{93970579}{870912} \zeta_{2} \log ^{2} 2-\frac{2057}{1152} N_{F} \zeta_{4} \log 2+\frac{63767}{2304} \zeta_{4} \log 2 \\
& -\frac{211}{10368} N_{F}^{3} \zeta_{3}+\frac{270407}{8957952} N_{F}^{3}-\frac{4091305}{1990656} N_{F}^{2} \zeta_{3}+\frac{576757}{331776} N_{F}^{2} \zeta_{4}+\frac{115}{2304} N_{F}^{2} \zeta_{5} \\
& +\frac{48073}{165888} N_{F}^{2}+\frac{151369}{4354560} N_{F} X_{0}+\frac{12171659669}{232243200} N_{F} \zeta_{3}-\frac{608462731}{69672960} N_{F} \zeta_{4} \\
& -\frac{313489}{41472} N_{F} \zeta_{5}-\frac{75861299783}{3135283200} N_{F}-\frac{4692439}{8709120} X_{0}-\frac{4660543511}{19353600} \zeta_{3} \\
& \left.-\frac{4674213853}{17418240} \zeta_{4}+\frac{807193}{10368} \zeta_{5}+\frac{846138861149}{3135283200}\right) L_{t}+\left(-\frac{481}{62208} N_{F}^{3}-\frac{28297}{110592} N_{F}^{2} \zeta_{3}\right. \\
& \left.+\frac{373637}{746496} N_{F}^{2}+\frac{2985893}{331776} N_{F} \zeta_{3}-\frac{47813}{4608} N_{F}-\frac{26296585}{442368} \zeta_{3}+\frac{143939741}{1990656}\right) L_{t}^{2} \\
& +\left(\frac{77}{124416} N_{F}^{3}+\frac{175}{27648} N_{F}^{2}-\frac{5855}{124416} N_{F}-\frac{130201}{124416}\right) L_{t}^{3} \\
& +\left(-\frac{1}{2592} N_{F}^{3}+\frac{47}{4608} N_{F}^{2}-\frac{317}{6912} N_{F}-\frac{51383}{165888}\right) L_{t}^{4}
\end{aligned}
$$

where the logarithms of the coefficient $C_{5}$ have been reconstructed from the result of refs. [68, 69] including the recent five-loop result of the QCD beta function [71] (partly confirmed by [72]). The constant $C_{50}$ is irrelevant for our derivation of the effective Lagrangian for gluonic Higgs couplings. Note that the highest powers of the logarithmic $L_{t}$ terms disappeared in this expression as required by the proper RG-evolution of 
the one-particle-irreducible part $\Pi_{t}$. Using the low-energy theorem for a light Higgs boson [52-54] the effective top-quark contribution to the Lagrangian of eq. (2.1) is related to the couplings of external Higgs bosons in the heavy-top-quark limit by the replacement ${ }^{2}$ $\overline{m_{t}}\left(\mu_{R}^{2}\right) \rightarrow \overline{m_{t}}\left(\mu_{R}^{2}\right)(1+H / v)$, i.e.

$$
L_{t} \rightarrow \bar{L}_{t}=L_{t}-2 \log \left(1+\frac{H}{v}\right) \quad \text { and } \quad \Pi_{t} \rightarrow \bar{\Pi}_{t}
$$

where $H$ denotes the physical Higgs field, $v$ the vacuum expectation value and $\bar{\Pi}_{t}$ the contribution to the Wilson coefficient with the shifted top-quark mass. ${ }^{3}$ Based on this replacement it is obvious that only the logarithmic $L_{t}$ terms of $\Pi_{t}$ are relevant for the effective gluonic Higgs couplings. The object $\bar{\Pi}_{t}$ is expressed in terms of the $\left(N_{F}+1\right)$ flavour coupling $\alpha_{s}^{\left(N_{F}+1\right)}$. To derive the low-energy Lagrangian in the $N_{F}$-flavour theory we have to transform the $\left(N_{F}+1\right)$-flavour coupling into the $N_{F}$-flavour one by means of the relation $[66-69]$

$$
\begin{aligned}
\alpha_{s}^{\left(N_{F}+1\right)}\left(\mu_{R}^{2}\right)= & \alpha_{s}^{\left(N_{F}\right)}\left(\mu_{R}^{2}\right)\left\{1+\frac{\alpha_{s}^{\left(N_{F}\right)}\left(\mu_{R}^{2}\right)}{\pi} \frac{L_{t}}{6}+\left(\frac{\alpha_{s}^{\left(N_{F}\right)}\left(\mu_{R}^{2}\right)}{\pi}\right)^{2}\left[-\frac{11}{72}+\frac{11}{24} L_{t}+\frac{L_{t}^{2}}{36}\right]\right. \\
& +\left(\frac{\alpha_{s}^{\left(N_{F}\right)}\left(\mu_{R}^{2}\right)}{\pi}\right)^{3}\left[-\frac{564731}{124416}+\frac{82043}{27648} \zeta_{3}+\frac{2633}{31104} N_{F}\right. \\
& \left.\left.+\left(\frac{2645}{1728}-\frac{67}{576} N_{F}\right) L_{t}+\left(\frac{167}{576}+\frac{N_{F}}{36}\right) L_{t}^{2}+\frac{L_{t}^{3}}{216}\right]+\mathcal{O}\left(\alpha_{s}^{4}\right)\right\}
\end{aligned}
$$

derived from inverting eq. (2.2). For the proper low-energy limit the gluonic field-strength operator is expressed in terms of the one with $N_{F}=5$ active flavours which leads to a global factor $\zeta_{\alpha_{s}}$ so that the kinetic term of the gluons is properly normalized in the lowenergy limit. ${ }^{4}$ In this way we arrive at the low-energy Lagrangian in terms of the top $\overline{\mathrm{MS}}$ mass. The effective $\mathrm{N}^{4} \mathrm{LO}$ Lagrangian for (multi-)Higgs couplings to gluons reads finally

$$
\begin{aligned}
\mathcal{L}_{\text {eff }}=\frac{\alpha_{s}}{12 \pi}\{(1+ & \delta) \log \left(1+\frac{H}{v}\right)-\frac{\eta}{2} \log ^{2}\left(1+\frac{H}{v}\right) \\
& \left.+\frac{\rho}{3} \log ^{3}\left(1+\frac{H}{v}\right)-\frac{\sigma}{4} \log ^{4}\left(1+\frac{H}{v}\right)\right\} G^{a \mu \nu} G_{\mu \nu}^{a}
\end{aligned}
$$

\footnotetext{
${ }^{2}$ In the case of an extended Higgs sector with several scalar Higgs bosons coupling to the top quark the replacement $\overline{m_{t}}\left(\mu_{R}^{2}\right) \rightarrow \overline{m_{t}}\left(\mu_{R}^{2}\right)\left(1+\sum_{i} c_{i} H_{i} / v\right)$ has to be implemented, where $c_{i}$ are the top quark Yukawa couplings normalized to the SM coupling. This results in the correspondence $H / v \leftrightarrow \sum_{i} c_{i} H_{i} / v$ for all subsequent steps.

${ }^{3}$ Note that diagrammatically for the single-Higgs case this expression coincides with the replacement $\frac{1}{\not p-m_{t}} \rightarrow \frac{1}{p-m_{t}} \frac{m_{t}}{v} \frac{1}{\not p-m_{t}}$ of the top-quark propagators inside the gluonic correlation functions up to 4th order in the gluon fields at the point where $m_{t}$ is either the unrenormalized or the pure $\overline{\mathrm{MS}}$ mass [28].

${ }^{4}$ Diagrammatically this step corresponds to adding the external $\overline{\mathrm{MS}}$-renormalized self-energies and twopoint-function contributions to the vertices involving top quarks at vanishing external momentum.
} 
with the QCD corrections up to $\mathrm{N}^{4} \mathrm{LO}$

$$
\begin{aligned}
\delta & =\delta_{1} \frac{\alpha_{s}}{\pi}+\delta_{2}\left(\frac{\alpha_{s}}{\pi}\right)^{2}+\delta_{3}\left(\frac{\alpha_{s}}{\pi}\right)^{3}+\delta_{4}\left(\frac{\alpha_{s}}{\pi}\right)^{4}+\mathcal{O}\left(\alpha_{s}^{5}\right) \\
\eta & =\eta_{2}\left(\frac{\alpha_{s}}{\pi}\right)^{2}+\eta_{3}\left(\frac{\alpha_{s}}{\pi}\right)^{3}+\eta_{4}\left(\frac{\alpha_{s}}{\pi}\right)^{4}+\mathcal{O}\left(\alpha_{s}^{5}\right) \\
\rho & =\rho_{3}\left(\frac{\alpha_{s}}{\pi}\right)^{3}+\rho_{4}\left(\frac{\alpha_{s}}{\pi}\right)^{4}+\mathcal{O}\left(\alpha_{s}^{5}\right) \\
\sigma & =\sigma_{4}\left(\frac{\alpha_{s}}{\pi}\right)^{4}+\mathcal{O}\left(\alpha_{s}^{5}\right)
\end{aligned}
$$

The explicit perturbative coefficients are given by

$$
\begin{aligned}
\delta_{1}= & \frac{11}{4} \\
\delta_{3}= & \frac{897943}{9216} \zeta_{3}-\frac{2892659}{41472}+\frac{209}{64} L_{t}^{2}+\frac{1733}{288} L_{t} \\
& +N_{F}\left(\frac{40291}{20736}-\frac{110779}{13824} \zeta_{3}+\frac{23}{32} L_{t}^{2}+\frac{55}{54} L_{t}\right)+N_{F}^{2}\left(-\frac{L_{t}^{2}}{18}+\frac{77}{1728} L_{t}-\frac{6865}{31104}\right) \\
\delta_{4}= & -\frac{121}{1440} N_{F} \log ^{5} 2+\frac{3751}{2880} \log ^{5} 2+\frac{685}{41472} N_{F}^{2} \log ^{4} 2+\frac{11679301}{1741824} N_{F} \log ^{4} 2 \\
& -\frac{93970579}{870912} \log ^{4} 2+\frac{121}{144} N_{F} \zeta_{2} \log ^{3} 2-\frac{3751}{288} \zeta_{2} \log ^{3} 2-\frac{685}{6912} N_{F}^{2} \zeta_{2} \log ^{2} 2 \\
& -\frac{11679301}{290304} N_{F} \zeta_{2} \log ^{2} 2+\frac{93970579}{145152} \zeta_{2} \log ^{2} 2+\frac{2057}{192} N_{F} \zeta_{4} \log 2-\frac{63767}{384} \zeta_{4} \log 2 \\
& +\frac{685}{1728} N_{F}^{2} a_{4}+\frac{11679301}{72576} N_{F} a_{4}-\frac{93970579}{36288} a_{4}+\frac{121}{12} N_{F} a_{5}-\frac{3751}{24} a_{5}+\frac{211}{1728} N_{F}^{3} \zeta_{3} \\
& -\frac{270407}{1492992} N_{F}^{3}+\frac{4091305}{331776} N_{F}^{2} \zeta_{3}-\frac{576757}{55296} N_{F}^{2} \zeta_{4}-\frac{115}{384} N_{F}^{2} \zeta_{5}-\frac{48073}{27648} N_{F}^{2} \\
& -\frac{151369}{725760} N_{F} X_{0}-\frac{12171659669}{38707200} N_{F} \zeta_{3}+\frac{608462731}{11612160} N_{F} \zeta_{4}+\frac{313489}{6912} N_{F} \zeta_{5} \\
& +\frac{76094378783}{522547200} N_{F}+\frac{4692439}{1451520} X_{0}+\frac{28121193841}{19353600} \zeta_{3}+\frac{4674213853}{2903040} \zeta_{4}-\frac{807193}{1728} \zeta_{5} \\
& -\frac{854201072999}{522547200}+\left(\frac{481}{5184} N_{F}^{3}+\frac{28297}{9216} N_{F}^{2} \zeta_{3}-\frac{21139}{3456} N_{F}^{2}-\frac{32257}{288} N_{F} \zeta_{3}\right. \\
& \left.+\frac{5160073}{41472} N_{F}+\frac{9364157}{12288} \zeta_{3}-\frac{49187545}{55296}\right) L_{t}+\left(-\frac{77}{6912} N_{F}^{3}-\frac{1267}{13824} N_{F}^{2}+\frac{4139}{2304} N_{F}\right. \\
& \left.+\frac{8401}{384}\right) L_{t}^{2}+\left(\frac{1}{108} N_{F}^{3}-\frac{157}{576} N_{F}^{2}+\frac{275}{192} N_{F}+\frac{2299}{256}\right) L_{t}^{3} \\
&
\end{aligned}
$$

and

$$
\begin{aligned}
& \eta_{2}=\frac{35}{24}+\frac{2}{3} N_{F} \\
& \eta_{3}=\frac{1333}{432}+\frac{589}{48} L_{t}+N_{F}\left(\frac{1081}{432}+\frac{191}{72} L_{t}\right)+N_{F}^{2}\left(\frac{77}{864}-\frac{2}{9} L_{t}\right)
\end{aligned}
$$




$$
\begin{aligned}
\eta_{4}= & \frac{481}{2592} N_{F}^{3}+N_{F}^{2}\left(\frac{28297}{4608} \zeta_{3}-\frac{373637}{31104}\right)+N_{F}\left(\frac{429965}{1728}-\frac{2985893}{13824} \zeta_{3}\right) \\
& +\frac{26296585}{18432} \zeta_{3}-\frac{143976701}{82944}+\left(-\frac{77}{1728} N_{F}^{3}-\frac{1421}{3456} N_{F}^{2}+\frac{9073}{1728} N_{F}+\frac{45059}{576}\right) L_{t} \\
& +\left(\frac{N_{F}^{3}}{18}-\frac{455}{288} N_{F}^{2}+\frac{63}{8} N_{F}+\frac{6479}{128}\right) L_{t}^{2} \\
\rho_{3}= & \frac{1697}{144}+\frac{175}{72} N_{F}-\frac{2}{9} N_{F}^{2} \\
\rho_{4}= & \frac{130201}{1728}+\frac{18259}{192} L_{t}+N_{F}\left(\frac{5855}{1728}+\frac{2077}{144} L_{t}\right)-N_{F}^{2}\left(\frac{175}{384}+\frac{439}{144} L_{t}\right) \\
& +N_{F}^{3}\left(\frac{L_{t}}{9}-\frac{77}{1728}\right) \\
\sigma_{4}= & \frac{51383}{864}+\frac{317}{36} N_{F}-\frac{47}{24} N_{F}^{2}+\frac{2}{27} N_{F}^{3}
\end{aligned}
$$

where $G_{\mu \nu}^{a}$ denotes the gluon field strength tensor and $\alpha_{s}$ the strong coupling constant with $N_{F}=5$ active flavours. Note that in accordance with the RG-evolution the coefficients $\delta_{1}, \eta_{2}, \rho_{3}$ and $\sigma_{4}$ are free of $L_{t}$ terms. Numerically we obtain for $N_{F}=5$ light flavours

$$
\begin{array}{ll}
\delta_{1}=2.75 & \delta_{2}=6.1528+2.8542 L_{t} \\
\delta_{3}=3.4043+12.2240 L_{t}+5.4705 L_{t}^{2} & \\
\delta_{4}=36.0373-73.5997 L_{t}+27.1760 L_{t}^{2}+10.4851 L_{t}^{3} & \\
\eta_{2}=4.7917 & \eta_{3}=17.8252+19.9792 L_{t} \\
\eta_{4}=-167.5239+88.6311 L_{t}+57.4401 L_{t}^{2} & \rho_{3}=18.3819 \\
\rho_{4}=75.3261+104.8906 L_{t} & \sigma_{4}=63.7998
\end{array}
$$

If the running $\overline{\mathrm{MS}}$ top mass is replaced by the top pole mass $M_{t}[73-78]^{5}$ [i.e. $L_{t}=\log \left(\mu_{R}^{2} / M_{t}^{2}\right)$ is used everywhere],

$$
\begin{aligned}
\bar{m}_{t}\left(\mu_{R}^{2}\right)=M_{t}\{ & 1-\left(\frac{4}{3}+\log \frac{\mu_{R}^{2}}{M_{t}^{2}}\right) \frac{\alpha_{s}^{\left(N_{F}\right)}\left(\mu_{R}^{2}\right)}{\pi}+\left[-\frac{3019}{288}-2 \zeta_{2}-\frac{2}{3} \zeta_{2} \log 2+\frac{\zeta_{3}}{6}\right. \\
& -\frac{461}{72} \log \frac{\mu_{R}^{2}}{M_{t}^{2}}-\frac{23}{24} \log ^{2} \frac{\mu_{R}^{2}}{M_{t}^{2}}+N_{F}\left(\frac{71}{144}+\frac{\zeta_{2}}{3}+\frac{13}{36} \log \frac{\mu_{R}^{2}}{M_{t}^{2}}+\frac{1}{12} \log ^{2} \frac{\mu_{R}^{2}}{M_{t}^{2}}\right) \\
& \left.\left.-\frac{4}{3} \sum_{1 \leq i \leq N_{F}} \Delta\left(\frac{M_{i}}{M_{t}}\right)\right]\left(\frac{\alpha_{s}^{\left(N_{F}\right)}\left(\mu_{R}^{2}\right)}{\pi}\right)^{2}\right\}+\mathcal{O}\left(\alpha_{s}^{3}\right)
\end{aligned}
$$

where the mass-dependent term involving the light flavours can (for $0 \leq x \leq 1$ ) be approximated by

$$
\Delta(x)=\frac{\pi^{2}}{8} x-0.579 x^{2}+0.230 x^{3}
$$

\footnotetext{
${ }^{5}$ Note that the low-energy strong coupling constant with $N_{F}=5$ active flavours is used in this relation.
} 
the QCD corrections are formally different from the $\overline{\mathrm{MS}}$ case above only for the coefficients $\delta_{3}, \delta_{4}$ and $\eta_{4}$,

$$
\begin{aligned}
& \delta_{3}=\frac{897943}{9216} \zeta_{3}-\frac{2761331}{41472}+\frac{209}{64} L_{t}^{2}+\frac{2417}{288} L_{t} \\
& +N_{F}\left(\frac{58723}{20736}-\frac{110779}{13824} \zeta_{3}+\frac{23}{32} L_{t}^{2}+\frac{91}{54} L_{t}\right)+N_{F}^{2}\left(-\frac{L_{t}^{2}}{18}+\frac{77}{1728} L_{t}-\frac{6865}{31104}\right) \\
& \delta_{4}=-\frac{121}{1440} N_{F} \log ^{5} 2+\frac{3751}{2880} \log ^{5} 2+\frac{685}{41472} N_{F}^{2} \log ^{4} 2+\frac{11679301}{1741824} N_{F} \log ^{4} 2 \\
& -\frac{93970579}{870912} \log ^{4} 2+\frac{121}{144} N_{F} \zeta_{2} \log ^{3} 2-\frac{3751}{288} \zeta_{2} \log ^{3} 2-\frac{685}{6912} N_{F}^{2} \zeta_{2} \log ^{2} 2 \\
& -\frac{11679301}{290304} N_{F} \zeta_{2} \log ^{2} 2+\frac{93970579}{145152} \zeta_{2} \log ^{2} 2+\frac{4}{9} N_{F} \zeta_{2} \log 2+\frac{19}{12} \zeta_{2} \log 2 \\
& +\frac{2057}{192} N_{F} \zeta_{4} \log 2-\frac{63767}{384} \zeta_{4} \log 2+\frac{685}{1728} N_{F}^{2} a_{4}+\frac{11679301}{72576} N_{F} a_{4}-\frac{93970579}{36288} a_{4} \\
& +\frac{121}{12} N_{F} a_{5}-\frac{3751}{24} a_{5}+\frac{211}{1728} N_{F}^{3} \zeta_{3}-\frac{270407}{1492992} N_{F}^{3}-\frac{2}{9} N_{F}^{2} \zeta_{2}+\frac{4091305}{331776} N_{F}^{2} \zeta_{3} \\
& -\frac{576757}{55296} N_{F}^{2} \zeta_{4}-\frac{115}{384} N_{F}^{2} \zeta_{5}-\frac{161627}{82944} N_{F}^{2}-\frac{151369}{725760} N_{F} X_{0}+\frac{13}{24} N_{F} \zeta_{2}+\frac{19}{4} \zeta_{2} \\
& -\frac{12175960469}{38707200} N_{F} \zeta_{3}+\frac{608462731}{11612160} N_{F} \zeta_{4}+\frac{313489}{6912} N_{F} \zeta_{5}+\frac{80863176383}{522547200} N_{F} \\
& +\frac{4692439}{1451520} X_{0}+\frac{28113533041}{19353600} \zeta_{3}+\frac{4674213853}{2903040} \zeta_{4}-\frac{807193}{1728} \zeta_{5}-\frac{831703495799}{522547200} \\
& +\left(\frac{481}{5184} N_{F}^{3}+\frac{28297}{9216} N_{F}^{2} \zeta_{3}-\frac{22687}{3456} N_{F}^{2}-\frac{32257}{288} N_{F} \zeta_{3}+\frac{5581849}{41472} N_{F}+\frac{9364157}{12288} \zeta_{3}\right. \\
& \left.-\frac{46543033}{55296}\right) L_{t}+\left(-\frac{77}{6912} N_{F}^{3}-\frac{5107}{13824} N_{F}^{2}+\frac{12547}{2304} N_{F}+\frac{14747}{384}\right) L_{t}^{2} \\
& +\left(\frac{1}{108} N_{F}^{3}-\frac{157}{576} N_{F}^{2}+\frac{275}{192} N_{F}+\frac{2299}{256}\right) L_{t}^{3}+\frac{4}{3}\left(\frac{2}{3} N_{F}+\frac{19}{8}\right) \sum_{1 \leq i \leq N_{F}} \Delta\left(\frac{M_{i}}{M_{t}}\right) \\
& \eta_{4}=\frac{481}{2592} N_{F}^{3}+N_{F}^{2}\left(\frac{28297}{4608} \zeta_{3}-\frac{392069}{31104}\right)+N_{F}\left(\frac{442189}{1728}-\frac{2985893}{13824} \zeta_{3}\right) \\
& +\frac{26296585}{18432} \zeta_{3}-\frac{141262589}{82944}+\left(-\frac{77}{1728} N_{F}^{3}-\frac{2957}{3456} N_{F}^{2}+\frac{18241}{1728} N_{F}+\frac{59195}{576}\right) L_{t} \\
& +\left(\frac{N_{F}^{3}}{18}-\frac{455}{288} N_{F}^{2}+\frac{63}{8} N_{F}+\frac{6479}{128}\right) L_{t}^{2}
\end{aligned}
$$

For the on-shell top-quark mass we obtain numerically for $N_{F}=5$ light flavours

$$
\begin{aligned}
& \delta_{3}=11.0154+17.9323 L_{t}+5.4705 L_{t}^{2} \\
& \delta_{4}=125.7997+13.8777 L_{t}+55.0041 L_{t}^{2}+10.4851 L_{t}^{3}+7.6111 \sum_{1 \leq i \leq N_{F}} \Delta\left(\frac{M_{i}}{M_{t}}\right) \\
& \eta_{4}=-114.2461+128.5894 L_{t}+57.4401 L_{t}^{2}
\end{aligned}
$$

The explicit expansion of the Lagrangian of eq. (2.8) in powers of the Higgs field results in

$$
\mathcal{L}_{\text {eff }}=\frac{\alpha_{s}}{12 \pi}\left\{\sum_{n=1}^{\infty} \Delta_{n} \frac{(-1)^{n-1}}{n}\left(\frac{H}{v}\right)^{n}\right\} G^{a \mu \nu} G_{\mu \nu}^{a}
$$


with the QCD corrections up to $\mathrm{N}^{4} \mathrm{LO}$

$$
\begin{aligned}
\Delta_{1}= & 1+\delta_{1} \frac{\alpha_{s}}{\pi}+\delta_{2}\left(\frac{\alpha_{s}}{\pi}\right)^{2}+\delta_{3}\left(\frac{\alpha_{s}}{\pi}\right)^{3}+\delta_{4}\left(\frac{\alpha_{s}}{\pi}\right)^{4}+\mathcal{O}\left(\alpha_{s}^{5}\right) \\
\Delta_{2}= & 1+\delta_{1} \frac{\alpha_{s}}{\pi}+\left(\delta_{2}+\eta_{2}\right)\left(\frac{\alpha_{s}}{\pi}\right)^{2}+\left(\delta_{3}+\eta_{3}\right)\left(\frac{\alpha_{s}}{\pi}\right)^{3}+\left(\delta_{4}+\eta_{4}\right)\left(\frac{\alpha_{s}}{\pi}\right)^{4}+\mathcal{O}\left(\alpha_{s}^{5}\right) \\
\Delta_{3}= & +\delta_{1} \frac{\alpha_{s}}{\pi}+\left(\delta_{2}+\frac{3}{2} \eta_{2}\right)\left(\frac{\alpha_{s}}{\pi}\right)^{2}+\left(\delta_{3}+\frac{3}{2} \eta_{3}+\rho_{3}\right)\left(\frac{\alpha_{s}}{\pi}\right)^{3} \\
& +\left(\delta_{4}+\frac{3}{2} \eta_{4}+\rho_{4}\right)\left(\frac{\alpha_{s}}{\pi}\right)^{4}+\mathcal{O}\left(\alpha_{s}^{5}\right) \\
\Delta_{4}= & +\delta_{1} \frac{\alpha_{s}}{\pi}+\left(\delta_{2}+\frac{11}{6} \eta_{2}\right)\left(\frac{\alpha_{s}}{\pi}\right)^{2}+\left(\delta_{3}+\frac{11}{6} \eta_{3}+2 \rho_{3}\right)\left(\frac{\alpha_{s}}{\pi}\right)^{3} \\
& +\left(\delta_{4}+\frac{11}{6} \eta_{4}+2 \rho_{4}+\sigma_{4}\right)\left(\frac{\alpha_{s}}{\pi}\right)^{4}+\mathcal{O}\left(\alpha_{s}^{5}\right) \\
\Delta_{5}= & 1+\delta_{1} \frac{\alpha_{s}}{\pi}+\left(\delta_{2}+\frac{25}{12} \eta_{2}\right)\left(\frac{\alpha_{s}}{\pi}\right)^{2}+\left(\delta_{3}+\frac{25}{12} \eta_{3}+\frac{35}{12} \rho_{3}\right)\left(\frac{\alpha_{s}}{\pi}\right)^{3} \\
& +\left(\delta_{4}+\frac{25}{12} \eta_{4}+\frac{35}{12} \rho_{4}+\frac{5}{2} \sigma_{4}\right)\left(\frac{\alpha_{s}}{\pi}\right)^{4}+\mathcal{O}\left(\alpha_{s}^{5}\right)
\end{aligned}
$$

for up to five external Higgs bosons. It should be noted that the coefficients $\delta_{1-4}$ of the single-Higgs term $\Delta_{1}$ agree with previous results up to $\mathrm{N}^{4} \mathrm{LO}$ [37, 38, 67-69, 79, 80], while the coefficient $\eta_{2}$ of the double-Higgs contribution $\Delta_{2}$ agrees with the explicit diagrammatic calculation of ref. [58].

Connecting our approach to derive the effective Lagrangian to the method of refs. [67-69] for the single-Higgs case we can easily derive their final relation,

$$
C_{H}=-\frac{1}{4} \zeta_{\alpha_{s}} g_{t} \partial_{m_{t}} \frac{1}{\zeta_{\alpha_{s}}}=\frac{1}{2 v} \frac{m_{t}^{2} \partial}{\partial\left(m_{t}^{2}\right)} \log \zeta_{\alpha_{s}}
$$

with $g_{t}=m_{t} / v, \partial_{m_{t}}=\partial / \partial m_{t}$ and $C_{H}$ denoting the full coefficient in front of the operator $G^{a \mu \nu} G_{\mu \nu}^{a} H$. This expression agrees with refs. [67-69]. For the double-Higgs case we arrive at

$$
C_{H H}=\frac{1}{8} \zeta_{\alpha_{s}} g_{t}^{2} \partial_{m_{t}}^{2} \frac{1}{\zeta_{\alpha_{s}}}=\frac{1}{4 v^{2}}\left\{\left(\frac{m_{t} \partial_{m_{t}} \zeta_{\alpha_{s}}}{\zeta_{\alpha_{s}}}\right)^{2}-\frac{m_{t}^{2} \partial_{m_{t}}^{2} \zeta_{\alpha_{s}}}{2 \zeta_{\alpha_{s}}}\right\}
$$

where $C_{H H}$ denotes the coefficient in front of the operator $G^{a \mu \nu} G_{\mu \nu}^{a} H^{2}$.

A final comment addresses the removal of one-particle-reducible contributions in eq. (2.4): this corresponds to the removal of one-particle-reducible diagrams of the type shown in figure 1 after attaching external Higgs bosons according to eq. (2.6). We have checked this correspondence explicitly for Higgs boson pair production in the heavy-topquark limit at NLO [55]. 


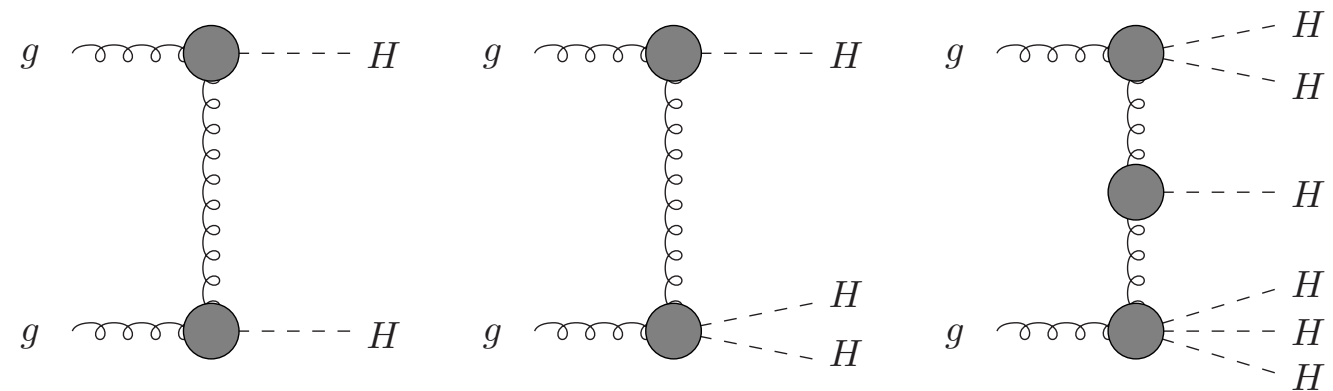

Figure 1. Typical one-particle-reducible Feynman diagrams for multi-Higgs boson production.

\section{Several heavy quarks}

Starting from the expression of the effective single-Higgs coupling to gluons of ref. [81] with $N_{H}$ heavy quarks contributing we can reconstruct the corresponding logarithmic parts of the function $\Pi_{Q}$,

$$
\begin{aligned}
\mathcal{L}_{g} & =-\frac{1-\Pi_{Q}}{4} \hat{G}^{a \mu \nu} \hat{G}_{\mu \nu}^{a} \\
\Pi_{Q} & =\sum_{n} C_{n}\left(\frac{\alpha_{s}^{\left(N_{F}+N_{H}\right)}}{\pi}\right)^{n}
\end{aligned}
$$

with the perturbative coefficients up to third order

$$
\begin{aligned}
& C_{1}=-\frac{N_{H}}{6} L_{Q} \\
& C_{2}=N_{H}\left[\frac{11}{72}-\frac{11}{24} L_{Q}\right] \\
& C_{3}=C_{30}-N_{H}\left(\frac{1877}{1152}-\frac{77}{3456} N_{H}-\frac{67}{576} N_{F}\right) L_{Q}-N_{H}\left(\frac{19}{192}-\frac{11}{288} N_{H}+\frac{N_{F}}{36}\right) L_{Q}^{2}
\end{aligned}
$$

where $\hat{G}^{a \mu \nu}$ denotes the gluonic field-strength operator of colour-SU(3) in the low-energy limit with $N_{F}+N_{H}$ active flavours. The logarithm is defined as

$$
L_{Q}=\frac{1}{N_{H}} \sum_{i=1}^{N_{H}} \log \left(\frac{\mu_{R}^{2}}{M_{i}^{2}}\right)
$$

For the derivation of the effective Lagrangian for the gluonic Higgs coupling the constant $C_{30}$ is irrelevant. Performing the replacement ${ }^{6}$

$$
L_{Q} \rightarrow \bar{L}_{Q}=L_{Q}-2 \log \left(1+\frac{H}{v}\right) \quad \text { and } \quad \Pi_{Q} \rightarrow \bar{\Pi}_{Q}
$$

\footnotetext{
${ }^{6}$ Here we assume SM-type couplings of the heavy quarks to the Higgs boson as e.g. for a sequential 4 th fermion generation. For the case of different couplings and $N_{S}$ scalar Higgs bosons this shift has to be replaced by $\log (1+H / v) \rightarrow \sum_{i=1}^{N_{H}} \log \left(1+\sum_{j=1}^{N_{S}} c_{i j} H_{j} / v\right) / N_{H}$ in all subsequent steps, where the factors $c_{i j}$ denote the Higgs Yukawa couplings normalized to the SM-Higgs coupling.
} 
and decoupling the heavy quarks from the strong coupling constant $\alpha_{s}$ by

$$
\begin{aligned}
\alpha_{s}^{\left(N_{F}+N_{H}\right)}\left(\mu_{R}^{2}\right)= & \alpha_{s}^{\left(N_{F}\right)}\left(\mu_{R}^{2}\right)\left\{1+\frac{\alpha_{s}^{\left(N_{F}\right)}\left(\mu_{R}^{2}\right)}{\pi} N_{H} \frac{L_{Q}}{6}\right. \\
& \left.+\left(\frac{\alpha_{s}^{\left(N_{F}\right)}\left(\mu_{R}^{2}\right)}{\pi}\right)^{2} N_{H}\left[-\frac{11}{72}+\frac{11}{24} L_{Q}+N_{H} \frac{L_{Q}^{2}}{36}\right]\right\}+\mathcal{O}\left(\alpha_{s}^{4}\right)
\end{aligned}
$$

and from the gluon-field-strength operator we arrive at the effective Lagrangian for the gluonic Higgs couplings up to NNLO

$$
\mathcal{L}_{\text {eff }}=N_{H} \frac{\alpha_{s}}{12 \pi}\left\{(1+\delta) \log \left(1+\frac{H}{v}\right)-\frac{\eta}{2} \log ^{2}\left(1+\frac{H}{v}\right)\right\} G^{a \mu \nu} G_{\mu \nu}^{a}
$$

with the QCD corrections up to NNLO

$$
\begin{aligned}
& \delta=\delta_{1} \frac{\alpha_{s}}{\pi}+\delta_{2}\left(\frac{\alpha_{s}}{\pi}\right)^{2}+\mathcal{O}\left(\alpha_{s}^{3}\right) \\
& \eta=\eta_{2}\left(\frac{\alpha_{s}}{\pi}\right)^{2}+\mathcal{O}\left(\alpha_{s}^{3}\right)
\end{aligned}
$$

The explicit perturbative coefficients read

$$
\begin{aligned}
& \delta_{1}=\frac{11}{4} \\
& \delta_{2}=\frac{1877}{192}-\frac{77}{576} N_{H}+\frac{19}{16} L_{Q}+N_{F}\left(\frac{L_{Q}}{3}-\frac{67}{96}\right) \\
& \eta_{2}=\frac{19}{8}-\frac{11}{12} N_{H}+\frac{2}{3} N_{F}
\end{aligned}
$$

The result for $\delta_{2}$ in the single-Higgs case agrees with the results of refs. [81, 82]. The NNLO results for more than one external Higgs boson are new.

\section{Conclusions}

In this work we have derived effective (multi-)Higgs couplings to gluons after integrating out all heavy quarks mediating these couplings. The effective Lagrangians can be used for the computation of the production of one or several Higgs bosons in gluon fusion at hadron colliders in the limit of heavy quarks. In the SM we have extended the effective Lagrangian for double-Higgs couplings to gluons to $\mathrm{N}^{4} \mathrm{LO}$ and derived for the first time the $\mathrm{N}^{4}$ LO Lagrangian for more than two SM Higgs bosons. In the second part we extended the analysis to the case of several heavy quarks coupling to the Higgs bosons up to NNLO. We reproduced the existing NNLO results for the single-Higgs case. We have derived these effective Lagrangians from their connection to the decoupling relations of the strong coupling constant.

\section{Acknowledgments}

We are grateful to M. Mühlleitner and A. Signer for carefully reading the manuscript and useful comments. This work is supported in part by the Research Executive Agency (REA) of the European Union under the Grant No. PITN-GA-2012-316704 (Higgstools). 
Open Access. This article is distributed under the terms of the Creative Commons Attribution License (CC-BY 4.0), which permits any use, distribution and reproduction in any medium, provided the original author(s) and source are credited.

\section{References}

[1] ATLAS collaboration, Observation of a new particle in the search for the Standard Model Higgs boson with the ATLAS detector at the LHC, Phys. Lett. B 716 (2012) 1 [arXiv: 1207.7214] [INSPIRE].

[2] CMS collaboration, Observation of a new boson at a mass of $125 \mathrm{GeV}$ with the CMS experiment at the LHC, Phys. Lett. B 716 (2012) 30 [arXiv:1207.7235] [INSPIRE].

[3] ATLAS, CMS collaborations, Measurements of the Higgs boson production and decay rates and constraints on its couplings from a combined ATLAS and CMS analysis of the LHC pp collision data at $\sqrt{s}=7$ and $8 \mathrm{TeV}$, JHEP 08 (2016) 045 [arXiv:1606.02266] [INSPIRE].

[4] P.W. Higgs, Broken symmetries, massless particles and gauge fields, Phys. Lett. 12 (1964) 132 [InSPIRE].

[5] P.W. Higgs, Broken Symmetries and the Masses of Gauge Bosons, Phys. Rev. Lett. 13 (1964) 508 [INSPIRE].

[6] P.W. Higgs, Spontaneous Symmetry Breakdown without Massless Bosons, Phys. Rev. 145 (1966) 1156 [INSPIRE].

[7] F. Englert and R. Brout, Broken Symmetry and the Mass of Gauge Vector Mesons, Phys. Rev. Lett. 13 (1964) 321 [INSPIRE].

[8] G.S. Guralnik, C.R. Hagen and T.W.B. Kibble, Global Conservation Laws and Massless Particles, Phys. Rev. Lett. 13 (1964) 585 [inSPIRE].

[9] G. 't Hooft, Renormalizable Lagrangians for Massive Yang-Mills Fields, Nucl. Phys. B 35 (1971) 167 [InSPIRE].

[10] G. 't Hooft and M.J.G. Veltman, Regularization and Renormalization of Gauge Fields, Nucl. Phys. B 44 (1972) 189 [inSPIRE].

[11] H.M. Georgi, S.L. Glashow, M.E. Machacek and D.V. Nanopoulos, Higgs Bosons from Two Gluon Annihilation in Proton Proton Collisions, Phys. Rev. Lett. 40 (1978) 692 [InSPIRE].

[12] A. Djouadi, M. Spira and P.M. Zerwas, Production of Higgs bosons in proton colliders: QCD corrections, Phys. Lett. B 264 (1991) 440 [INSPIRE].

[13] S. Dawson, Radiative corrections to Higgs boson production, Nucl. Phys. B 359 (1991) 283 [INSPIRE].

[14] S. Dawson and R. Kauffman, QCD corrections to Higgs boson production: nonleading terms in the heavy quark limit, Phys. Rev. D 49 (1994) 2298 [hep-ph/9310281] [INSPIRE].

[15] R.V. Harlander and W.B. Kilgore, Soft and virtual corrections to $p p \rightarrow H+X$ at $N N L O$, Phys. Rev. D 64 (2001) 013015 [hep-ph/0102241] [InSPIRE].

[16] R.V. Harlander and W.B. Kilgore, Next-to-next-to-leading order Higgs production at hadron colliders, Phys. Rev. Lett. 88 (2002) 201801 [hep-ph/0201206] [INSPIRE].

[17] C. Anastasiou and K. Melnikov, Higgs boson production at hadron colliders in NNLO QCD, Nucl. Phys. B 646 (2002) 220 [hep-ph/0207004] [INSPIRE]. 
[18] V. Ravindran, J. Smith and W.L. van Neerven, NNLO corrections to the total cross-section for Higgs boson production in hadron hadron collisions, Nucl. Phys. B 665 (2003) 325 [hep-ph/0302135] [INSPIRE].

[19] S. Marzani, R.D. Ball, V. Del Duca, S. Forte and A. Vicini, Higgs production via gluon-gluon fusion with finite top mass beyond next-to-leading order, Nucl. Phys. B 800 (2008) 127 [arXiv:0801.2544] [INSPIRE].

[20] T. Gehrmann, M. Jaquier, E.W.N. Glover and A. Koukoutsakis, Two-Loop QCD Corrections to the Helicity Amplitudes for $H \rightarrow 3$ partons, JHEP 02 (2012) 056 [arXiv:1112.3554] [INSPIRE].

[21] C. Anastasiou, C. Duhr, F. Dulat and B. Mistlberger, Soft triple-real radiation for Higgs production at N3LO, JHEP 07 (2013) 003 [arXiv: 1302.4379] [INSPIRE].

[22] C. Anastasiou, C. Duhr, F. Dulat, F. Herzog and B. Mistlberger, Real-virtual contributions to the inclusive Higgs cross-section at $N^{3} L O$, JHEP 12 (2013) 088 [arXiv:1311.1425] [INSPIRE].

[23] W.B. Kilgore, One-loop single-real-emission contributions to $p p \rightarrow H+X$ at next-to-next-to-next-to-leading order, Phys. Rev. D 89 (2014) 073008 [arXiv:1312.1296] [INSPIRE].

[24] Y. Li, A. von Manteuffel, R.M. Schabinger and H.X. Zhu, Soft-virtual corrections to Higgs production at $N^{3} L O$, Phys. Rev. D 91 (2015) 036008 [arXiv:1412.2771] [INSPIRE].

[25] C. Anastasiou et al., Higgs boson gluon-fusion production beyond threshold in $N^{3} L O Q C D$, JHEP 03 (2015) 091 [arXiv:1411.3584] [INSPIRE].

[26] C. Anastasiou, C. Duhr, F. Dulat, F. Herzog and B. Mistlberger, Higgs Boson Gluon-Fusion Production in QCD at Three Loops, Phys. Rev. Lett. 114 (2015) 212001 [arXiv:1503.06056] [INSPIRE].

[27] C. Anastasiou et al., High precision determination of the gluon fusion Higgs boson cross-section at the LHC, JHEP 05 (2016) 058 [arXiv: 1602.00695] [INSPIRE].

[28] M. Spira, A. Djouadi, D. Graudenz and P.M. Zerwas, Higgs boson production at the LHC, Nucl. Phys. B 453 (1995) 17 [hep-ph/9504378] [INSPIRE].

[29] D. Graudenz, M. Spira and P.M. Zerwas, QCD corrections to Higgs boson production at proton proton colliders, Phys. Rev. Lett. 70 (1993) 1372 [INSPIRE].

[30] R. Harlander and P. Kant, Higgs production and decay: Analytic results at next-to-leading order QCD, JHEP 12 (2005) 015 [hep-ph/0509189] [INSPIRE].

[31] C. Anastasiou, S. Bucherer and Z. Kunszt, HPro: A NLO Monte-Carlo for Higgs production via gluon fusion with finite heavy quark masses, JHEP 10 (2009) 068 [arXiv:0907.2362] [INSPIRE].

[32] R.V. Harlander and K.J. Ozeren, Top mass effects in Higgs production at next-to-next-to-leading order QCD: Virtual corrections, Phys. Lett. B 679 (2009) 467 [arXiv: 0907.2997] [INSPIRE].

[33] R.V. Harlander and K.J. Ozeren, Finite top mass effects for hadronic Higgs production at next-to-next-to-leading order, JHEP 11 (2009) 088 [arXiv:0909.3420] [INSPIRE].

[34] A. Pak, M. Rogal and M. Steinhauser, Virtual three-loop corrections to Higgs boson production in gluon fusion for finite top quark mass, Phys. Lett. B 679 (2009) 473 [arXiv: 0907.2998] [INSPIRE]. 
[35] A. Pak, M. Rogal and M. Steinhauser, Finite top quark mass effects in NNLO Higgs boson production at LHC, JHEP 02 (2010) 025 [arXiv:0911.4662] [INSPIRE].

[36] R. Mueller and D.G. Öztürk, On the computation of finite bottom-quark mass effects in Higgs boson production, JHEP 08 (2016) 055 [arXiv: 1512.08570] [INSPIRE].

[37] M. Krämer, E. Laenen and M. Spira, Soft gluon radiation in Higgs boson production at the LHC, Nucl. Phys. B 511 (1998) 523 [hep-ph/9611272] [INSPIRE].

[38] M. Spira, QCD effects in Higgs physics, Fortsch. Phys. 46 (1998) 203 [hep-ph/9705337] [INSPIRE].

[39] S. Catani, D. de Florian, M. Grazzini and P. Nason, Soft gluon resummation for Higgs boson production at hadron colliders, JHEP 07 (2003) 028 [hep-ph/0306211] [INSPIRE].

[40] S. Moch and A. Vogt, Higher-order soft corrections to lepton pair and Higgs boson production, Phys. Lett. B 631 (2005) 48 [hep-ph/0508265] [INSPIRE].

[41] V. Ravindran, On Sudakov and soft resummations in QCD, Nucl. Phys. B 746 (2006) 58 [hep-ph/0512249] [INSPIRE].

[42] V. Ravindran, Higher-order threshold effects to inclusive processes in QCD, Nucl. Phys. B 752 (2006) 173 [hep-ph/0603041] [INSPIRE].

[43] A. Idilbi, X.-d. Ji, J.-P. Ma and F. Yuan, Threshold resummation for Higgs production in effective field theory, Phys. Rev. D 73 (2006) 077501 [hep-ph/0509294] [INSPIRE].

[44] V. Ahrens, T. Becher, M. Neubert and L.L. Yang, Renormalization-Group Improved Prediction for Higgs Production at Hadron Colliders, Eur. Phys. J. C 62 (2009) 333 [arXiv: 0809.4283] [INSPIRE].

[45] D. de Florian and M. Grazzini, Higgs production through gluon fusion: Updated cross sections at the Tevatron and the LHC, Phys. Lett. B 674 (2009) 291 [arXiv:0901.2427] [InSPIRE].

[46] D. de Florian, J. Mazzitelli, S. Moch and A. Vogt, Approximate $N^{3}$ LO Higgs-boson production cross section using physical-kernel constraints, JHEP 10 (2014) 176 [arXiv: 1408.6277] [INSPIRE].

[47] S. Catani, L. Cieri, D. de Florian, G. Ferrera and M. Grazzini, Threshold resummation at $N^{3} L L$ accuracy and soft-virtual cross sections at $N^{3} L O$, Nucl. Phys. B 888 (2014) 75 [arXiv: 1405.4827] [INSPIRE].

[48] M. Bonvini and L. Rottoli, Three loop soft function for $N^{3} L L^{\prime}$ gluon fusion Higgs production in soft-collinear effective theory, Phys. Rev. D 91 (2015) 051301 [arXiv:1412.3791] [INSPIRE].

[49] D. de Florian and M. Grazzini, Higgs production at the LHC: updated cross sections at $\sqrt{s}=8$ TeV, Phys. Lett. B 718 (2012) 117 [arXiv:1206.4133] [INSPIRE].

[50] M. Bonvini and S. Marzani, Resummed Higgs cross section at $N^{3} L L$, JHEP 09 (2014) 007 [arXiv: 1405.3654] [INSPIRE].

[51] T. Schmidt and M. Spira, Higgs Boson Production via Gluon Fusion: Soft-Gluon Resummation including Mass Effects, Phys. Rev. D 93 (2016) 014022 [arXiv:1509.00195] [INSPIRE].

[52] J.R. Ellis, M.K. Gaillard and D.V. Nanopoulos, A Phenomenological Profile of the Higgs Boson, Nucl. Phys. B 106 (1976) 292 [InSPIRE]. 
[53] M.A. Shifman, A.I. Vainshtein, M.B. Voloshin and V.I. Zakharov, Low-Energy Theorems for Higgs Boson Couplings to Photons, Sov. J. Nucl. Phys. 30 (1979) 711 [inSPIRE].

[54] B.A. Kniehl and M. Spira, Low-energy theorems in Higgs physics, Z. Phys. C 69 (1995) 77 [hep-ph/9505225] [INSPIRE].

[55] S. Dawson, S. Dittmaier and M. Spira, Neutral Higgs boson pair production at hadron colliders: QCD corrections, Phys. Rev. D 58 (1998) 115012 [hep-ph/9805244] [INSPIRE].

[56] D. de Florian and J. Mazzitelli, Two-loop virtual corrections to Higgs pair production, Phys. Lett. B 724 (2013) 306 [arXiv:1305.5206] [INSPIRE].

[57] D. de Florian and J. Mazzitelli, Higgs Boson Pair Production at Next-to-Next-to-Leading Order in QCD, Phys. Rev. Lett. 111 (2013) 201801 [arXiv:1309.6594] [INSPIRE].

[58] J. Grigo, K. Melnikov and M. Steinhauser, Virtual corrections to Higgs boson pair production in the large top quark mass limit, Nucl. Phys. B 888 (2014) 17 [arXiv:1408.2422] [INSPIRE].

[59] D.Y. Shao, C.S. Li, H.T. Li and J. Wang, Threshold resummation effects in Higgs boson pair production at the LHC, JHEP 07 (2013) 169 [arXiv:1301.1245] [INSPIRE].

[60] D. de Florian and J. Mazzitelli, Higgs pair production at next-to-next-to-leading logarithmic accuracy at the LHC, JHEP 09 (2015) 053 [arXiv: 1505.07122] [INSPIRE].

[61] S. Borowka et al., Higgs Boson Pair Production in Gluon Fusion at Next-to-Leading Order with Full Top-Quark Mass Dependence, Phys. Rev. Lett. 117 (2016) 012001 [Erratum ibid. 117 (2016) 079901] [arXiv: 1604.06447] [INSPIRE].

[62] S. Borowka et al., Full top quark mass dependence in Higgs boson pair production at NLO, arXiv: 1608.04798 [INSPIRE].

[63] J. Grigo, J. Hoff, K. Melnikov and M. Steinhauser, On the Higgs boson pair production at the LHC, Nucl. Phys. B $\mathbf{8 7 5}$ (2013) 1 [arXiv: 1305.7340] [INSPIRE].

[64] R. Frederix et al., Higgs pair production at the LHC with NLO and parton-shower effects, Phys. Lett. B 732 (2014) 142 [arXiv:1401.7340] [INSPIRE].

[65] F. Maltoni, E. Vryonidou and M. Zaro, Top-quark mass effects in double and triple Higgs production in gluon-gluon fusion at NLO, JHEP 11 (2014) 079 [arXiv:1408.6542] [INSPIRE].

[66] W. Bernreuther and W. Wetzel, Decoupling of Heavy Quarks in the Minimal Subtraction Scheme, Nucl. Phys. B 197 (1982) 228 [Erratum ibid. B 513 (1998) 758] [INSPIRE].

[67] K.G. Chetyrkin, B.A. Kniehl and M. Steinhauser, Decoupling relations to $O\left(\alpha_{s}^{3}\right)$ and their connection to low-energy theorems, Nucl. Phys. B 510 (1998) 61 [hep-ph/9708255] [INSPIRE].

[68] Y. Schröder and M. Steinhauser, Four-loop decoupling relations for the strong coupling, JHEP 01 (2006) 051 [hep-ph/0512058] [INSPIRE].

[69] K.G. Chetyrkin, J.H. Kuhn and C. Sturm, QCD decoupling at four loops, Nucl. Phys. B 744 (2006) 121 [hep-ph/0512060] [InSPIRE].

[70] Y. Schröder and A. Vuorinen, High-precision $\epsilon$-expansions of single-mass-scale four-loop vacuum bubbles, JHEP 06 (2005) 051 [hep-ph/0503209] [INSPIRE].

[71] P.A. Baikov, K.G. Chetyrkin and J.H. Kühn, Five-Loop Running of the QCD coupling constant, arXiv: 1606.08659 [INSPIRE]. 
[72] T. Luthe, A. Maier, P. Marquard and Y. Schröder, Towards the five-loop $\beta$-function for a general gauge group, JHEP 07 (2016) 127 [arXiv: 1606.08662] [INSPIRE].

[73] N. Gray, D.J. Broadhurst, W. Grafe and K. Schilcher, Three Loop Relation of Quark (Modified) Ms and Pole Masses, Z. Phys. C 48 (1990) 673 [InSPIRE].

[74] K.G. Chetyrkin and M. Steinhauser, Short distance mass of a heavy quark at order $\alpha_{s}^{3}$, Phys. Rev. Lett. 83 (1999) 4001 [hep-ph/9907509] [INSPIRE].

[75] K.G. Chetyrkin and M. Steinhauser, The relation between the MS-bar and the on-shell quark mass at order $\alpha_{s}^{3}$, Nucl. Phys. B 573 (2000) 617 [hep-ph/9911434] [InSPIRE].

[76] K. Melnikov and T.v. Ritbergen, The three loop relation between the MS-bar and the pole quark masses, Phys. Lett. B 482 (2000) 99 [hep-ph/9912391] [INSPIRE].

[77] P. Marquard, A.V. Smirnov, V.A. Smirnov and M. Steinhauser, Quark Mass Relations to Four-Loop Order in Perturbative QCD, Phys. Rev. Lett. 114 (2015) 142002 [arXiv: 1502.01030] [INSPIRE].

[78] A.L. Kataev and V.S. Molokoedov, On the flavour dependence of the $\mathcal{O}\left(\alpha_{s}^{4}\right)$ correction to the relation between running and pole heavy quark masses, Eur. Phys. J. Plus 131 (2016) 271 [arXiv: 1511.06898] [INSPIRE].

[79] T. Inami, T. Kubota and Y. Okada, Effective Gauge Theory and the Effect of Heavy Quarks in Higgs Boson Decays, Z. Phys. C 18 (1983) 69 [INSPIRE].

[80] K.G. Chetyrkin, B.A. Kniehl and M. Steinhauser, Hadronic Higgs decay to order $\alpha_{s}^{4}$, Phys. Rev. Lett. 79 (1997) 353 [hep-ph/9705240] [INSPIRE].

[81] A.G. Grozin et al., Simultaneous decoupling of bottom and charm quarks, JHEP 09 (2011) 066 [arXiv:1107.5970] [InSPIRE].

[82] C. Anastasiou, R. Boughezal and E. Furlan, The NNLO gluon fusion Higgs production cross-section with many heavy quarks, JHEP 06 (2010) 101 [arXiv: 1003.4677] [INSPIRE]. 